\title{
Comparing the Efficacy of FeLV Vaccines
}

Comment on : Stuke, K. et al. Efficacy of an inactivated FeLV vaccine compared to a recombinant FeLV vaccine in minimum age cats following virulent FeLV challenge. Vaccine 2014;32(22):2599-603.

Dear Editor,

Having read with interest the study by Stuke et al. comparing the efficacy of two feline leukemia virus (FeLV) vaccines (Versifel and Purevax), we should like to bring several observations to your readers' attention, which are important enough to express doubt about the validity of the data and the authors' conclusions.

Intercurrent infection with feline parvovirus (FPV): During the vaccine experiment, an infection with FPV occurred. Two cats were recognized as being infected by FPV. Twenty other cats persistently viraemic with FeLV ( 7 belonging to the control and 13 belonging to the Purevax group) were euthanized before FeLV related clinical signs developed. In the paper, no information is provided regarding whether or not FPV was also present in the persistently viraemic cats and to what extent cats vaccinated by Versifel had been exposed to FPV. As the FeLV challenge virus contained FPV (as suggested by positive PCR results), it was concluded that the intercurrent infection had been caused by the inadvertent injection of FPV with the challenge virus. Accordingly, we must assume that all cats were exposed to both FeLV and FPV, rendering the validity of the data regarding vaccine efficacy questionable, and the title of the paper misleading. Parvoviruses are known to exhaust the bone marrow, reducing leukocyte counts ("panleukopenia"); they are highly immunosuppressive, and a study on the immunogenicity of a product cannot be considered valid under these conditions. It is not representative for the conditions under which vaccines are used in the field.

The published results give the impression that the Purevax vaccine is ineffective, which is at variance with earlier observations by independent researchers [1-3]. In the Discussion, the authors offer the possibility that the FPV infection may have "caused a lower than expected efficacy in the Purevax vaccine group...", ignoring the volume of knowledge about parvoviral immune suppression. It is a significant concern that the 
superficial reader will remember only the low vaccine efficacy and the poor performance of the recombinant vaccine.

The authors could have demonstrated that the intercurrent infection had indeed been caused by infectious FPV present in the challenge inoculum. Positive PCR results do not absolutely prove the presence of infectious virus. Other sources of an FPV infection may have played a role (e.g. contaminated cages or surfaces, toys that are introduced to provide environmental enrichment to cats kept in groups). Also, the authors do not provide information on the physical location of the three experimental groups: their spatial arrangement (was there direct contact?), attendance (did the animals share the same animal caretakers?), opportunity for indirect infection (were they exposed to fomites?).

Latent infection: The authors state that "latent infection ... can be detected in the bone marrow by PCR" and they cite two papers $(6,7)$ published in 1982 and 1983. At that time, the PCR technique invented by Kary Mullis in 1983 was not yet in the public domain. To date, FeLV PCR results obtained with bone marrow as specimen have not been shown to be a parameter for latent infection; the results of table 2 are therefore misleading. Indeed, it is difficult to ascertain the validity of the PCR data as no information is provided on the target sequences amplified by either the FeLV or the FPV PCR assays. Moreover, the authors provide spurious information on the qPCR using bone marrow which was "done according to established procedure at Biological Development, Zoetis. The method is described in (19), with slight modification in cycles used". Again, the cited reference is from 1981 (before the invention of PCR as a technique had been described) and contains no information about qPCR.

Experimental approach: Where the efficacy of two vaccine preparations are being compared, it is essential that the relatedness of the challenge strain and the vaccine strains be clearly defined. Stuke et al. provide no information on the infectious dose used to infect the cats and the relatedness between the FeLV strain used for vaccine production and the challenge virus. The challenge virus used in this experiment might have been homologous to the inactivated vaccine but heterologous to the recombinant vaccine. This aspect was not addressed by the authors in the Discussion.

We were concerned by the number of additional variables in the experiments that may have impacted on the outcome of the trial: Why it was necessary to 
prophylactically treat cats with sulfadimethoxine for coccidiosis? Why were male cats castrated halfway in the course of the experiment? Castration is a stressful intervention that influences immune reactions. The authors did not mention the number of male cats assigned to the different groups.

Why did the authors not collect blood samples in post-challenge weeks 1 and 2 . These are important time points, as some vaccines lead to early integration of FeLV provirus; this can readily be seen in the PCR results.

There are many additional minor flaws in the study by Stuke et al. which we would not expect to encounter in a learned journal such as "Vaccine". For example, FeLV is a gammaretrovirus and does not belong to the lentivirus family, it belongs to the Retroviridae. References are not cited correctly: references (1) and (2) do not describe "FeLV as one of the most important viral pathogens". Finally, the statement that "FeLVrelated clinical signs were observed in two animals..." is unwarranted - after all, neither FPV nor FeLV infection signs are pathognomonic.

While we appreciate that "Vaccine" cannot withdraw this paper; we respectively request that our objections to its validity should reach the Journal's readership.

Regina Hofmann-Lehmann, Laura S. Levy \& Brian Willett

[1] Poulet H, Brunet S, Boularand C, Guiot AL, Leroy V, Tartaglia J, et al. Efficacy of a canarypox virus-vectored vaccine against feline leukaemia. Vet Rec. 2003;153:141-5.

[2] Grosenbaugh DA, Leard T, Pardo MC. Protection from challenge following administration of a canarypox virus-vectored recombinant feline leukemia virus vaccine in cats previously vaccinated with a killed virus vaccine. J Am Vet Med Assoc. 2006;228:726-7.

[3] Grosenbaugh DA, Leard T, Pardo MC, Motes-Kreimeyer L, Royston M. Comparison of the safety and efficacy of a recombinant feline leukemia virus (FeLV) vaccine delivered transdermally and an inactivated FeLV vaccine delivered subcutaneously. Veterinary therapeutics : research in applied veterinary medicine. 2004;5:258-62. 